\title{
Proximal Resection Margins: More Prognostic than Distal Resection Margins in Patients Undergoing Hilar Cholangiocarcinoma Resection
}

\author{
Tae Yoo, MD 1 \\ Sang-Jae Park, MD, PhD² \\ Sung-Sik Han, MD, PhD² \\ Seong Hoon Kim, MD, PhD2 \\ Seung Duk Lee, MD, PhD² \\ Tae Hyun Kim, MD, PhD² \\ Soon-ae Lee, MD, $P h D^{2}$ \\ Sang Myung Woo, MD, PhD² \\ Woo Jin Lee, MD, PhD² \\ Eun Kyung Hong, MD, PhD²
}

${ }^{1}$ Department of Surgery, Hallym University College of Medicine, Hwaseong,

${ }^{2}$ Center for Liver Cancer,

National Cancer Center, Goyang, Korea
Correspondence: Sang-Jae Park, MD, PhD Center for Liver Cancer, National Cancer Center, 323 Ilsan-ro, Ilsandong-gu, Goyang 10408, Korea Tel: 82-31-920-1640

Fax: 82-31-920-2799

E-mail:spark@ncc.re.kr

Received July 3, 2017

Accepted November 15, 2017

Published Online November 16, 2017

\begin{abstract}
Purpose
Even though the therapeutic gold standard of hilar cholangiocarcinoma (HCCA) resection is cancer-free resection margin (RM), surgical treatment still remains challenging. This study evaluated the prognostic significance of RM status in resected HCCA patients and identified survival prognostic factors.
\end{abstract}

\section{Materials and Methods}

We reviewed records of 96 HCCA patients who underwent surgery from 2001 to 2012 and analyzed the RM status and prognostic factors that affecting survival.

\section{Results}

Negative RM ( $n=31,33 \%)$ was significantly associated with better survival vs. positive RM $(n=65,67 \%$ ) (mean survival time [MST], 33 months vs. 21 months; $p=0.011$ ). Margins with histological findings of non-dysplastic epithelium, low-grade dysplasia, and carcinoma in situ were not associated with survival differences (MST, 33 months vs. 33 months vs. 30 months; $p=0.452$ ), whereas positive margins were associated with poorer survival relative to carcinoma in situ (MST, 30 months vs. 21 months; $p=0.050$ ). Among patients with RO resection, narrow $(\leq 5 \mathrm{~mm})$ and wide $(>5 \mathrm{~mm})$ margins were not associated with survival differences (MST, 33 months vs. 30 months; $p=0.234$ ). Although positive proximal RM was associated with poorer survival compared to negative RM (MST, 19 vs. 33; $p=0.002$ ), no survival difference was observed between positive and negative distal RMs (MST, 30 vs. 33; $p=0.628$ ). Proximal RM positivity (hazard ratio [HR], 2.688; $p=0.007$ ) and nodal involvement (HR, 3.293; $p<0.001)$ were independent survival prognostic factors.

\section{Conclusion}

A clear RM, especially proximal RM status, was significant prognosticator, and proximal bile duct resection to the greatest technically feasible extent may be necessary, with careful consideration of the potential morbidity and oncologic outcomes after resection. However, an aggressive approach to obtain a negative distal RM might be controversial and should be considered carefully, depending on the patient's status.

\section{Introduction}

Hilar cholangiocarcinoma (HCCA) is an uncommon malignancy that affects the hepatic duct confluence and for which surgical treatment remains challenging. Currently, the therapeutic gold standard is complete macroscopic tumor resection, or curative resection, defined as the achievement
Key words

Hilar cholangiocarcinoma, Resection margin, Liver resection of histologically cancer-free resection margins (RM) [1,2]. Most centers have recently adopted protocols for R0 resection that comprise major hepatectomy combined with en bloc resection of the extrahepatic bile duct and caudate lobe, thus improving the likelihood of a cure, and selected patients have also undergone pancreatoduodenectomy to ensure complete resection of positive distal bile ducts [3,4]. Despite aggressive surgical therapy, the location and tendency of 
HCCA to infiltrate closed vasculature and hepatic tissue have maintained the difficulty of bile duct resection with histologically clear surgical margins [5]. Therefore, several reports of cases with positive margins have described the performance of additional resection to the extent of technically feasibility $[6,7]$. However, despite these efforts, relation between RM status and survival benefit is still not well known. Therefore, we conducted this study to evaluate the prognostic significance of resection margin status in patients who have undergone resection of HCCA and to identify the prognostic factors affecting patient survival.

\section{Materials and Methods}

Between 2001-2012, 117 consecutive patients with tumors involving the hepatic hilum underwent surgical interventions with curative intent; of these, 103 underwent tumor resection (resectability rate, $88.3 \%$ ). Seven of the latter were excluded from the analysis because they died within 30 days of surgery $(6.7 \%)$. Therefore, 96 patients were enrolled in this study. The 74 men and 22 women included in this study had a mean age of 61.8 years (range, 36 to 85 years), and all had adenocarcinoma arising from the hepatic ducts that involved the biliary confluence.

Preoperative diagnoses were based on a combination of radiologic imaging findings, tumor marker (carbohydrate antigen 19-9, carcinoembryonic antigen) analyses, and routine medical assessments. Degrees of tumor extension were assessed using dynamic computed tomography and/or dynamic magnetic resonance imaging, and the biliary anatomy and extent of biliary obstruction were evaluated using endoscopic or percutaneous cholangiography. Positron emission tomography was used to characterize cross-sectional radiographic findings indicating suspected metastatic disease. The longitudinal extents of resected tumors along the bile duct were classified using a modified Bismuth classification as type I $(\mathrm{n}=18,19 \%)$, II $(\mathrm{n}=15,15 \%)$, IIIa $(\mathrm{n}=22$, $23 \%), \mathrm{IIIb}(\mathrm{n}=12,12 \%)$, or IV $(\mathrm{n}=30,31 \%)$. Preoperative biliary drainage was performed in 86 patients (89\%). Endoscopic nasogastric biliary drainage $(\mathrm{n}=18,20 \%)$, endoscopic retrograde biliary drainage $(n=9,12 \%)$, or percutaneous transhepatic biliary drainage $(n=59,68 \%)$ was performed to reduce the cholestatic liver damage in cases with preoperative jaundice for a median of 20 days (range, 4 to 56 days). The median preoperative total bilirubin level after biliary drainage was $2.4 \mathrm{mg} / \mathrm{dL}$ (range, 0.3 to $9 \mathrm{mg} / \mathrm{dL}$ ). Portal vein embolization $(n=9,12 \%)$ of the liver segment to be resected was performed to induce compensatory hypertrophy of the future remnant liver if the estimated resection volume comprised $<30 \%-40 \%$ of the whole liver.

The potential for complete removal of tumoral disease was the major incentive for resection. All surgical resections included right hemihepatectomy $(\mathrm{n}=51,53 \%)$, right trisegmentectomy $(n=8,8 \%)$, left hemihepatectomy $(n=20,21 \%)$, left trisegmentectomy $(\mathrm{n}=2,2 \%)$, and/or hilar bile duct resection with or without caudate lobectomy $(n=10,11 \%)$. Pancreatoduodenectomy was also performed $(n=5,5 \%)$ if the tumor had invaded the pancreatic head. No patients underwent only bile duct resection. Routine lymphadenectomy included the dissection of lymph nodes located in the hepatoduodenal ligament, behind the pancreatic head, along the common hepatic artery, and at the right side of the celiac artery root. Portal vein resection and anastomosis were conducted in cases involving portal vein invasion $(n=9,12 \%)$. Frozen tissue sections were used for intraoperative pathological assessments of the proximal or distal bile duct transection lines. If safety margin was proved to be positive, addition hepatobiliary resection was done as far as technically feasible until R0 was obtained if possible. The mean operation time was 571 minutes (range, 340 to 900 minutes). Intraoperative transfusion was performed in 27 patients $(28 \%)$.

The extrahepatic bile duct was incised longitudinally from the distal to the proximal margin for gross tumor identification. Major bile ducts were serially cross-sectioned at 2-mm intervals to identify any invasive carcinoma components and to determine the depth of invasion. Specific attention was directed toward the deepest aspect of the tumor and its interfaces with the liver parenchyma, portal vein, hepatic artery branches, and perihilar soft tissue. The latter was thoroughly sectioned to examine radial margins and identify lymph nodes. The radial and ductal margins of all resected specimens were evaluated by one experienced pancreatobiliary pathologist. Histologically, RM statuses were classified as follows: margin with non-dysplastic epithelium (NDE; $\mathrm{n}=54,56 \%$ ), margin with low-grade dysplasia (LGD; $\mathrm{n}=5$, $5 \%$ ), margin with carcinoma in situ (CIS; $\mathrm{n}=6,6 \%$ ), or margin with invasive carcinoma $(n=31,33 \%)$. High-grade dysplasia was classified as CIS because these statuses were very difficult to distinguish. Surgical curability was defined by the residual tumor status: a negative $\mathrm{RM}(\mathrm{n}=65,67 \%)$ indicated a margin with NDE, LGD, or CIS, whereas a positive margin $(n=31,33 \%)$ indicated the existence of invasive carcinoma in the final pathologic review. Moreover, patients classed as R0 were further subdivided by negative margin length: the narrow margin group comprised those in whom the tumor was found $\leq 5 \mathrm{~mm}$ from the cut surface but was not exposed $(n=43,44 \%)$, whereas the wide margin group comprised those in whom the free margin was $>5 \mathrm{~mm}$ from the cut surface $(n=22,23 \%)$. Patients with positive RMs were also subclassified according to location: positive proximal margin 
only $(\mathrm{n}=20,21 \%)$, positive distal margin only $(\mathrm{n}=5,5 \%)$, and both positive proximal and distal margins $(n=6,6 \%)$. Eighteen patients with a positive RM (58\%) underwent additional resection surgeries. Given the technical challenges associated with additional resection (e.g., biliary and vascular anomalies, insufficient liver remnant, comorbidities), however, a negative RM could not be achieved in all 26 cases with positive RM, despite the performance of a maximal transection. For patients with a positive distal margin only, cancer-free margins were achieved by right-sided $(n=4)$ or left-sided hepatectomy $(\mathrm{n}=1)$ with caudate lobectomy in the proximal duct lesion. However, the positive margins remained. Five patients did not undergo an additional pancreaticoduodenectomy because of comorbidities such as chronic obstructive pulmonary disease $(n=1)$, congestive heart failure $(n=2)$, or poor nutritional and performance statuses $(\mathrm{n}=2)$, although all patients $(n=5)$ underwent additional resection surgery (i.e., intrapancreatic bile duct resection).

All tumor specimens were examined pathologically and classified as well , moderate or poorly differentiated adenocarcinoma based on the predominant pathological grading of differentiation. Perineural invasion, hepatic invasion, and lymph node metastasis were also examined. The final HCCA stage was determined pathologically using the International Union Against Cancer TNM classification system of malignant tumors, seventh edition [8]. After discharge, no patient was lost during a median follow-up period of 19 months. Adjuvant therapy was administered to patients with positive $\mathrm{RM}$ and/or nodal involvement according to the National Comprehensive Cancer Network guidelines.

\section{Statistical analysis}

Survival data of all patients were obtained from our institution's electronic/paper medical records. The Kaplan-Meier method of survival analysis was implemented, and the logrank test was used for group comparisons. Univariate correlations between clinicopathologic factors and cumulative survival were also examined using the log-rank test to identify potential prognostic factors, and a Cox proportional hazard regression analysis was used to determine factors independently associated with cumulative survival. All statistical analyses were performed using STATA ver. 10.1 for Windows (StataCorp., College Station, TX). All statistical test results were two-sided, and a p-value $<0.05$ was considered statistically significant.

\section{Ethical statement}

This retrospective study protocol was approved by the Institutional Review Board of the National Cancer Center, Korea (NCC2017-0044). The informed consent was waived.

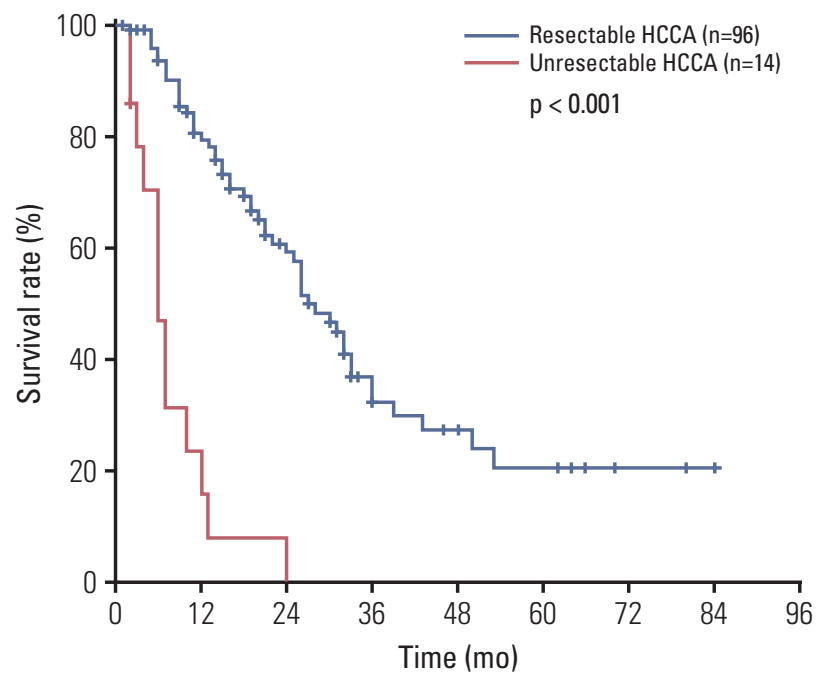

Fig. 1. Overall survival of hilar cholangiocarcinoma (HCCA) patients (resectable vs. unresectable).

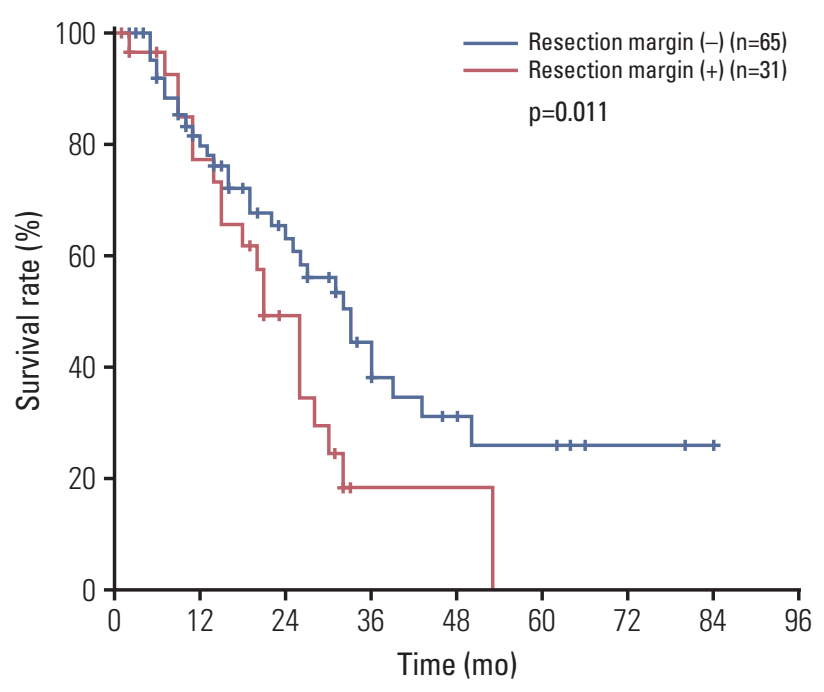

Fig. 2. Survival of hilar cholangiocarcinoma patients according to resection margin status.

\section{Results}

\section{Survival according to the resection margin status}

The overall median survival time (MST) among patients with resectable HCCA was 27 months (range, 2 to 84 months), and the estimated overall survival rates at 1,3 , and 


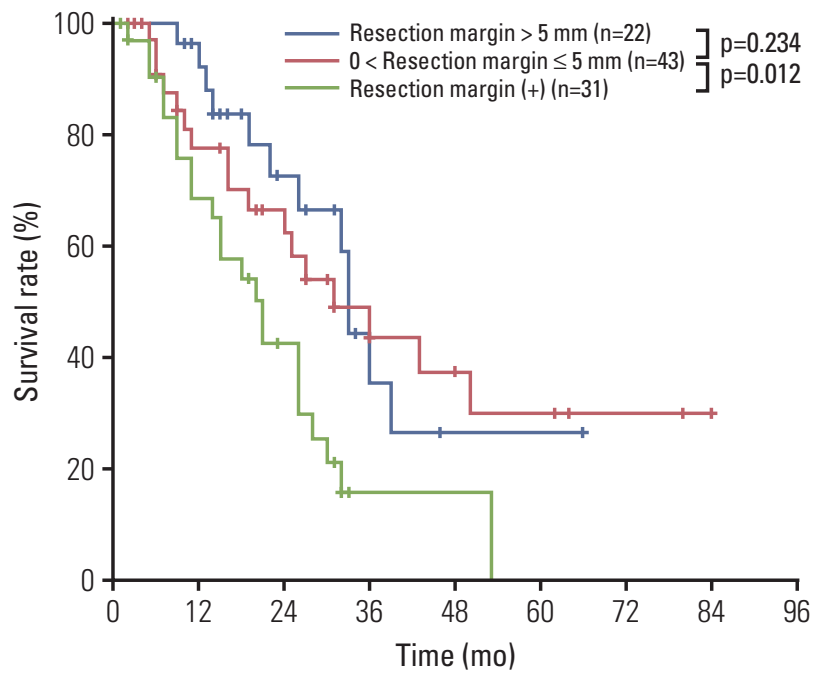

Fig. 3. Survival of hilar cholangiocarcinoma patients by surgical free margin length.

5 years were $79.2 \%, 32.1 \%$, and $20.4 \%$, respectively (Fig. 1). Fig. 2 presents a survival analysis according to residual tumor status. Of the 96 included patients, 65 (67\%) had negative RMs and 31 (33\%) had positive RMs, and the former exhibited better survival relative to the latter (MST, 33 months vs. 21 months; $\mathrm{p}=0.011$ ). We also evaluated survival by margin histology and observed no differences among patients whose margins included NDE, LGD, and CIS (MST, 33 months vs. 33 months vs. 30 months; $p=0.452$ ). However, patients with positive margins (i.e., invasive carcinoma) had worse survival outcomes than did those whose margins contained CIS (MST, 30 months vs. 21 months; $\mathrm{p}=0.050$ ).

\section{Surgical free margin length}

Fig. 3 demonstrates survival according to surgical free margins. Among the 65 patients who underwent R0, 43 (44\%) had narrow surgical margins and $22(23 \%)$ had wide margins. We observed no difference in survival after $\mathrm{R} 0$ resection between these groups (MST, 33 months and 30 months, respectively; $\mathrm{p}=0.234$ ). However, the narrow margin group achieved significantly better survival than did the positive RM group (MST, 30 months vs. 21 months; $\mathrm{p}=0.012$ ).

\section{Positive resection margin location}

Among the 31 patients with positive RMs, 20 (21\%) had positive proximal RMs only, and five ( $5 \%$ ) had positive distal RM only. In our survival analysis (Fig. 4), patients with positive proximal margins had worse survival outcomes than

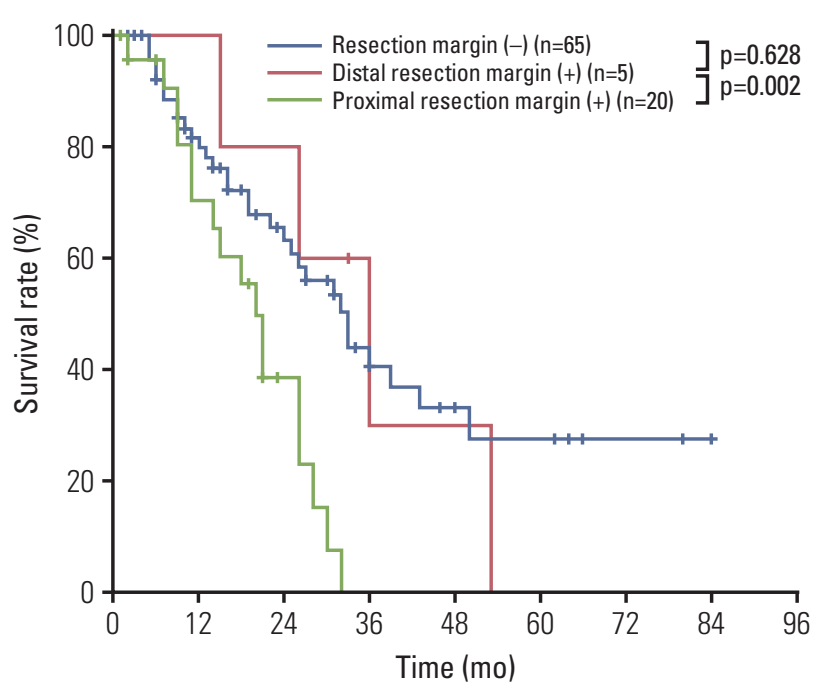

Fig. 4. Survival of hilar cholangiocarcinoma patients by positive resection margin location.

did those with negative RMs (MST, 19 months vs. 33 months; $\mathrm{p}=0.002$ ). However, we observed no significant survival difference between those with positive distal margins and those with negative proximal and distal margins (MST, 30 months vs. 33 months; $\mathrm{p}=0.628$ ).

\section{Analysis of prognostic factors}

We analyzed the prognostic significance of 18 clinicopathologic variables (Table 1). The univariate analysis revealed that resection margin status (MST, 33 months vs. 21 months; $\mathrm{p}=0.011$ ), proximal RM positivity (MST, 33 months vs. 19 months; $\mathrm{p}=0.002)$, lymph node involvement (MST, 32 months vs. 15 months; $\mathrm{p}<0.001$ ), adjuvant therapy (MST, 33 months vs. 21 months; $\mathrm{p}=0.01$ ), and CA 19-9 level (MST, 32 months vs. 21 months; $\mathrm{p}=0.022$ ) were significantly associated with patient survival. A multivariate analysis confirmed that proximal RM positivity (hazard ratio [HR], 2.688; $\mathrm{p}=0.007$ ) and lymph node involvement $(\mathrm{HR}, 3.293$; $\mathrm{p}<0.001)$ remained independently associated with survival.

\section{Discussion}

HCCA is still considered a disease with a dismal prognosis, and its local infiltrative characteristics and close continuity to the main portal vein and its branches, as well as the hepatic artery, render surgical resection a significant action. 
Table 1. Univariate and multivariate analyses of clinicopathologic factors for overall survival

\begin{tabular}{|c|c|c|c|c|c|}
\hline Factor & No. & $\begin{array}{c}\text { Median } \\
\text { survival (mo) }\end{array}$ & $\begin{array}{c}\text { HR } \\
(95 \% \mathrm{CI})\end{array}$ & $\begin{array}{c}\text { Univariate } \\
\text { p-value }\end{array}$ & $\begin{array}{c}\text { Multivariate } \\
\text { p-value }\end{array}$ \\
\hline \multicolumn{6}{|l|}{ Sex } \\
\hline Male & 21 & 22 & - & 0.444 & - \\
\hline Female & 75 & 27 & & & \\
\hline \multicolumn{6}{|l|}{ Age (yr) } \\
\hline$<60$ & 61 & 28 & - & 0.242 & - \\
\hline$\geq 60$ & 35 & 25 & & & \\
\hline \multicolumn{6}{|l|}{ Bismuth type } \\
\hline I/II & $18 / 15$ & $33 / 32$ & - & 0.188 & - \\
\hline III/ IV & $33 / 30$ & $27 / 24$ & & & \\
\hline \multicolumn{6}{|l|}{ Biliary drainages } \\
\hline Yes & 86 & 36 & - & 0.140 & - \\
\hline No & 10 & 32 & & & \\
\hline \multicolumn{6}{|l|}{ CA $19-9(\mathrm{U} / \mathrm{mL})$} \\
\hline$<37$ & 29 & 36 & 3.437 & 0.022 & 0.064 \\
\hline$\geq 37$ & 67 & 22 & & & \\
\hline \multicolumn{6}{|l|}{ Operation time (min) } \\
\hline$<500$ & 46 & 26 & - & 0.340 & - \\
\hline$\geq 500$ & 50 & 26 & & & \\
\hline \multicolumn{6}{|l|}{ Transfusion } \\
\hline Yes & 27 & 26 & - & 0.087 & - \\
\hline No & 69 & 36 & & & \\
\hline Cell differentiation (well/moderate/poor) & $33 / 39 / 24$ & $32 / 39 / 21$ & - & 0.114 & - \\
\hline \multicolumn{6}{|l|}{ Tumor size $(\mathrm{cm})$} \\
\hline$<4$ & 40 & 30 & - & 0.105 & - \\
\hline$\geq 4$ & 51 & 26 & & & \\
\hline \multicolumn{6}{|l|}{ Perineural invasion } \\
\hline Yes & 25 & 25 & - & 0.394 & - \\
\hline No & 71 & 28 & & & \\
\hline \multicolumn{6}{|l|}{ Lymphatic invasion } \\
\hline Yes & 30 & 25 & - & 0.180 & - \\
\hline No & 67 & 36 & & & \\
\hline \multicolumn{6}{|l|}{ Microvascular invasion } \\
\hline Yes & 33 & 18 & - & 0.120 & - \\
\hline No & 63 & 30 & & & \\
\hline \multicolumn{6}{|l|}{ Resection margin } \\
\hline$(-)$ & 31 & 33 & 1.768 & 0.011 & 0.178 \\
\hline$(+)$ & 65 & 21 & & & \\
\hline \multicolumn{6}{|l|}{ Length of negative margin (mm) } \\
\hline$\geq 5$ & 22 & 33 & - & 0.234 & - \\
\hline$<5$ & 43 & 30 & & & \\
\hline \multicolumn{6}{|l|}{ Proximal margin status } \\
\hline$(+)$ & 20 & 19 & $\begin{array}{c}2.688 \\
(1.311-5.510)\end{array}$ & 0.002 & 0.007 \\
\hline$(-)$ & 65 & 33 & & & \\
\hline \multicolumn{6}{|l|}{ LN metastasis } \\
\hline Yes & 40 & 15 & $\begin{array}{c}3.293 \\
(1.686-6.434)\end{array}$ & $<0.001$ & $<0.001$ \\
\hline No & 56 & 32 & & & \\
\hline
\end{tabular}

(Continued to the next page) 
Table 1. Continued

\begin{tabular}{|c|c|c|c|c|c|}
\hline Factor & No. & $\begin{array}{c}\text { Median } \\
\text { survival (mo) }\end{array}$ & $\begin{array}{c}\text { HR } \\
(95 \% \mathrm{CI})\end{array}$ & $\begin{array}{l}\text { Univariate } \\
\text { p-value }\end{array}$ & $\begin{array}{c}\text { Multivariate } \\
\text { p-value }\end{array}$ \\
\hline \multicolumn{6}{|c|}{ Stage (UICC 7th) } \\
\hline I/II/III & $33 / 62 / 2$ & $33 / 26 / 24$ & & 0.128 & - \\
\hline \multicolumn{6}{|c|}{ Adjuvant therapy } \\
\hline Yes & 67 & 33 & 1.25 & 0.011 & 0.264 \\
\hline No & 29 & 21 & & & \\
\hline
\end{tabular}

HR, hazard ratio; CI, confidence interval; CA 19-9, carbohydrate antigen 19-9; LN, lymph node; UICC International Union Against Cancer.

Recent studies have proposed that aggressive surgery, including major hepatic resection combined with en bloc extrahepatic bile duct resection and lymph node dissection, is only the demonstrable factor associated with long-term patient survival. Jarnagin et al. [2] highlighted the importance of en bloc major hepatectomy in their study, where they demonstrated a survival benefit incurred by patients who underwent concomitant liver resection relative to those who underwent only excision of the extrahepatic biliary tree (MST, 42.9 months vs. 28.8 months; $\mathrm{p}=0.002$ ). Kondo et al. [9] observed that patients who underwent extended hepatectomy with resection of the biliary tree exhibited a quite remarkable median overall survival of 27 months and 3-year survival rate of $40 \%$. Likewise, Hemming et al. [10] achieved a median survival of 40 months after major hepatectomy. Our study regarding the routine performance of aggressive hepatic resection with bile duct resection corroborated those earlier reports, as we achieved a MST 27 months with a 3 -year survival rate of $32 \%$. Actually, hepatectomy combined with bile duct resection is mainly performed to increase the likelihood of margin-negative resection. The majority of reports indicate that a positive resection margin strongly affects prognosis and emphasize the importance of achieving a tumor-free RM. Hirano et al. [11] reported a correlation between the surgical margin status and survival, with 3-and 5 -year survival rates $(24.2 \%$ and $6 \%$, respectively) among patients with histologically positive margins that were significantly inferior to the corresponding rates ( $52 \%$ and $39.9 \%$, respectively) observed in patients with $\mathrm{R} 0$ resection $(p=0.009)$. Similarly, Seyama et al. [12] confirmed that survival differed significantly according to the bile duct margin status and reported 5-year survival rates of $46.1 \%, 34.7 \%$, and $0 \%$ after R0, R1, and R2 resections, respectively $(\mathrm{p}<0.001)$. The present study also revealed that patients with microscopically positive RM had significantly worse survival, compared to patients with clear margins (MST, 33 months vs. 21 months; $\mathrm{p}=0.011$ ). However, several authors have suggested that patients whose positive ductal margins contain carcinoma in situ could survive beyond 5 years. Shirai et al. [13] evaluated ductal margin statuses in patients with extrahepatic cholangiocarcinoma who underwent resection and demonstrated that invasive carcinoma, but not carcinoma in situ, in ductal RM had a strong adverse effect on patient survival. We also studied this issue and found similar results. In our study, we classified patients into four groups according to resection margin histology: negative margin, margin with LGD, CIS, and invasive carcinoma. The first three groups did not differ significantly with respect to survival, whereas those whose positive margins contained invasive carcinoma had worse survival outcomes than did those with CIS. Therefore, we defined CIS as a negative margin. We also examined surgical margin lengths in patients with $\mathrm{R} 0$ resection to analyze the influence of resection margin on survival outcome. Patients with narrow and wide negative RM exhibited similar survival patterns, whereas those who underwent R1 resection exhibited significantly worse survival outcomes compared to patients with narrow negative resection margins. Several previous studies have associated a positive RM status with an increased risk of worse survival. However, no differences in prognostic patterns were observed between patients with narrow $(\leq 5 \mathrm{~mm})$ vs. wide $(>5 \mathrm{~mm})$ resection margins. Murakami et al. [14] evaluated negative margin lengths in 28 cholangiocarcinoma patients who underwent R0 resection and found similar rates of recurrence among patients with margins $\leq 5 \mathrm{~mm}$ and $>5 \mathrm{~mm}(\mathrm{p}=0.930)$. A multicenter analysis including 16 centers and 434 patients recently showed that survival was not influenced by the length of a negative RM. In that study, the length of a negative margin appeared to have no long-term impact, as survival estimates of patients with a negative margin of 0.5-9 $\mathrm{mm}$ and those of patients with a negative margin $>1 \mathrm{~cm}$ were similar $(\mathrm{p}=0.610)$, and both were significantly better than those in patients with a positive margin $(\mathrm{p}=0.001)$ [15].

We further evaluated patient survival according to the location of the positive RM. Among our cases with proximal resection margins, 22 patients $(21 \%)$ had positive proximal 
RM only, and these patients had significantly worse survival outcomes than did those with negative margins. However, among cases with distal bile duct margins, we observed no significant difference in survival between the positive and negative duct margin groups.

We further identified a positive proximal surgical margin as one of the strongest independent risk factors for survival among patients with resected HCCA and note that although we assume that this finding is accurate, the effects of margin location (proximal vs. distal margin) have not been well studied. However, some studies were conducted to evaluate the prognostic relationship between RM location and survival in patients with extrahepatic bile duct cancer. One recent study by Konishi et al. [16] showed that a positive proximal ductal margin correlated significantly with poor survival (HR, 1.72; $\mathrm{p}=0.029)$ and anastomotic recurrence ( $\mathrm{HR}, 6.39 ; \mathrm{p}=0.008)$, but found that a positive distal margin was not significant in cases of extrahepatic cholangiocarcinoma. We attribute the differences in survival prognosis between patients with a positive distal RM and those with a positive proximal RM to anatomical differences in the cause of death. Tumor progression in the proximal bile duct margin generally causes sepsis due to cholangitis and hepatic failure. Here, tumor progression causes biliary strictures and obstruction of the hepaticojejunostomy site, leading to cholangitis. Furthermore, the stenosis of multiple bile ducts fails to control the infection, thus predisposing the patient to sepsis. In addition, residual tumor progression in the proximal duct involves the invasion of the surrounding blood vessels, leading to hepatic failure and associated complications. Therefore, patients with positive proximal margins tend to die from sepsis or liver failure before experiencing cancer progression. By contrast, the risk of biliary complications is somewhat lower among patients with a positive distal RM, even if the tumor has progressed.

Undoubtedly, the number of patients with a positive distal $\mathrm{RM}$ is too small to allow us to draw valid conclusions or conduct further statistical analyses. Furthermore, prospective studies involving larger numbers of enrolled patients are needed to confirm the results of the present study. However, the data presented herein suggest that the resection of HCCA is rare, as this tumor has a poor prognosis, and few papers have analyzed and categorized these cases according to the RM location. Therefore, it would be meaningful to evaluate prognostic tendencies according to the RM location.

Accordingly, if the proximal ductal margin is positive and the distal margin is negative, every reasonable attempt should be made to clear the proximal margin. However, in cases with positive distal RMs, the use of an aggressive approach to obtain a negative distal RM might be controversial and should be carefully considered according to each patient's status.

Our study was subject to some limitations. It was a retrospective study involving a limited number of patients. Moreover, by design, our study was based on more than 10 years of experience with HCCA treatment. Advances in surgical techniques and chemotherapy might have introduced an element of lead-time bias; in other words, patients treated more recently may have better survival rates because of lessons learned from increased experience, better adjuvant therapies, and better supportive care. Furthermore, most of our patients had advanced Klatskin-type tumors (Bismuth's classification types 3 and 4,65.6\%). Therefore, the applicability of our results may be limited. However, this issue is clinically important, and prospective studies enrolling larger numbers of patients are required to confirm the results of this study in future.

In conclusion, our findings demonstrate that achieving a clear resection margin is among the most important prognostic factors for survival in patients with resected HCCA, regardless of the surgical margin length, and identify proximal RM positivity as a significant independent risk factor for survival. Thus, bile duct resection to the greatest technically feasible extent may be necessary, with careful consideration of the potential morbidity and oncologic outcomes after resection. However, the use of an aggressive approach to obtain a negative distal RM might be controversial and should be carefully considered according to each patient's status.

\section{Conflicts of Interest}

Conflict of interest relevant to this article was not reported.

\section{References}

1. Bismuth H, Nakache R, Diamond T. Management strategies in resection for hilar cholangiocarcinoma. Ann Surg. 1992;215: 31-8.

2. Jarnagin WR, Ruo L, Little SA, Klimstra D, D'Angelica M, DeMatteo RP, et al. Patterns of initial disease recurrence after resection of gallbladder carcinoma and hilar cholangiocarcinoma: implications for adjuvant therapeutic strategies. Cancer. 2003;98:1689-700.

3. Dinant S, Gerhards MF, Busch OR, Obertop H, Gouma DJ, Van Gulik TM. The importance of complete excision of the 
caudate lobe in resection of hilar cholangiocarcinoma. HPB (Oxford). 2005;7:263-7.

4. Kawahara R, Tanigawa M, Horiuchi H, Takahashi K, Shirahama T, Nakama Y, et al. A case of postoperative recurrent hilar cholangiocarcinoma in the lower bile duct treated with pancreatoduodenectomy. Gan To Kagaku Ryoho. 2014;41: 1518-20.

5. Geng XP, Zhu HG, Liu FB, Hou H, Zhang ZG, Zhao HC, et al. Anatomy problems in the radical resection for hilar cholangiocarcinoma. Zhonghua Wai Ke Za Zhi. 2009;47:1167-70.

6. Shingu Y, Ebata T, Nishio H, Igami T, Shimoyama Y, Nagino $\mathrm{M}$. Clinical value of additional resection of a margin-positive proximal bile duct in hilar cholangiocarcinoma. Surgery. 2010;147:49-56.

7. Ribero D, Amisano M, Lo Tesoriere R, Rosso S, Ferrero A, Capussotti L. Additional resection of an intraoperative margin-positive proximal bile duct improves survival in patients with hilar cholangiocarcinoma. Ann Surg. 2011;254:776-81.

8. Zaydfudim VM, Clark CJ, Kendrick ML, Que FG, Reid-Lombardo KM, Donohue JH, et al. Correlation of staging systems to survival in patients with resected hilar cholangiocarcinoma. Am J Surg. 2013;206:159-65.

9. Kondo S, Hirano S, Ambo Y, Tanaka E, Okushiba S, Morikawa $\mathrm{T}$, et al. Forty consecutive resections of hilar cholangiocarcinoma with no postoperative mortality and no positive ductal margins: results of a prospective study. Ann Surg. 2004;240:
95-101.

10. Hemming AW, Reed AI, Fujita S, Foley DP, Howard RJ. Surgical management of hilar cholangiocarcinoma. Ann Surg. 2005;241:693-9.

11. Hirano S, Kondo S, Tanaka E, Shichinohe T, Tsuchikawa T, Kato K, et al. Outcome of surgical treatment of hilar cholangiocarcinoma: a special reference to postoperative morbidity and mortality. J Hepatobiliary Pancreat Sci. 2010;17:455-62.

12. Seyama Y, Kubota K, Sano K, Noie T, Takayama T, Kosuge T, et al. Long-term outcome of extended hemihepatectomy for hilar bile duct cancer with no mortality and high survival rate. Ann Surg. 2003;238:73-83.

13. Shirai Y, Sakata J, Wakai T, Hatakeyama K. Intraoperative assessment of the resectability of hilar cholangiocarcinoma. Hepatogastroenterology. 2012;59:2436-8.

14. Murakami S, Ajiki T, Okazaki T, Ueno K, Kido M, Matsumoto $\mathrm{I}$, et al. Factors affecting survival after resection of intrahepatic cholangiocarcinoma. Surg Today. 2014;44:1847-54.

15. Ribero D, Pinna AD, Guglielmi A, Ponti A, Nuzzo G, Giulini SM, et al. Surgical approach for long-term survival of patients with intrahepatic cholangiocarcinoma: a multi-institutional analysis of 434 patients. Arch Surg. 2012;147:1107-13.

16. Konishi M, Iwasaki M, Ochiai A, Hasebe T, Ojima H, Yanagisawa A. Clinical impact of intraoperative histological examination of the ductal resection margin in extrahepatic cholangiocarcinoma. Br J Surg. 2010;97:1363-8. 